\title{
Alternate Splicing
}

National Cancer Institute

\section{Source}

National Cancer Institute. Alternate Splicing. NCI Thesaurus. Code C16280.

Alternate Splicing is a process in which multiple protein isoforms or products are generated from a single gene as a result of splicing together different nonconsecutive exons during the processing of initially identical nascent transcripts of a gene into mature mRNA transcripts. An exon may be connected to any one of several alternative exons to form the mRNA and generate related proteins, often in a tissue or developmental stage specific manner. 